\title{
Chironomus gr. decorus (DIPTERA: CHIRONOMIDAE) E OUTROS INSETOS AQUÁTICOS DE UM AÇUDE TEMPORÁRIO DO SEMI-ÁRIDO PARAIBANO, BRASIL
}

\author{
Francisco José Pegado Abílio(1), Alaíde A. Fonseca-Gessner (2), \\ Takako Watanabe(1) \& Romualdo Lunguinho Leite ${ }^{(3)}$
}

\begin{abstract}
Chironomus gr. decorus (Diptera: Chironomidae) and anothers aquatic insects in a temporary dam from Paraiban semi-arid, Brazil The aim purpose was to study the composition of Chironomidae fauna and the anothers aquatic insects in a temporary dam. Qualitative samples were realized in two periods: August/1995, higher level of water and March/1999, after the completely drying dam for 3 years. Only one species of Chironomidae was registered, Chironomus gr. decorus, in both periods, which contributed with $28.09 \%$ for the total aquatic insects fauna in August/ 95 and it was the only species to colonize the sediment of dam after 3 years of drying period. In August/95 observed the occurrence of Odonata, Coleoptera, Hemiptera, Ephemeroptera and Trichoptera. In this period was evidenced lower values of dissolvid nitrogen (ammonium, nitrite and nitrate), sulfate and electric conductivity compared with March/99. Thus, the aquatic

(1) Departamento de Metodologia da Educação, Centro de Educação e Departamento de Sistemática e Ecologia/Centro de Ciências Exatas e da Natureza, Universidade Federal da Paraíba, Rua Maria Rosa Padilha, 84, Edifício Aeroville, Ap. 210, Bairro Bessa, João Pessoa, CEP: 58037-260 Paraiba. E-mail: chicopegado@hotmail.com; (2) Departamento de Hidrobiologia, Universidade Federal de São Carlos, Via Washington Luis, Km 235, Caixa Postal 676, CEP: 13565-905 São Carlos, São Paulo; (3) Faculdade de Filosofia Dom Aureliano Matos, Universidade Estadual do Ceará, Rua Jaime Leonel Chaves, 688, CEP: 63930-000 Centro, Limoeiro do Norte, Ceará.
\end{abstract}

Com apoio financeiro do CNPq e CAPES. 
insects fauna were strongly ifluenced by physical and chemycal conditions. Two considerations its may be registred, in spite of appearing of Chironomus gr. decorus, immediately after the dry period: 1 . The oviposition by flying females, originating from permanent waters, is the most important means of recolonizing habitat refilled; 2 . Or the species it may be survive the desiccation, as larvae in dry sediment or in cocoon during the dry period.

Key words: Chironomidae, Chironomus gr. decorus, semi-arid, temporay dam

\section{Resumo}

Com o objetivo de estudar a composição da fauna de Chironomidae e de outros insetos aquáticos do açude temporário Soledade, Soledade-PB (07은 $02^{\prime} \mathrm{S}$ e $\left.36^{\circ} 22^{\prime} \mathrm{W}\right)$, coletas qualitativas foram realizadas em dois períodos: agosto de 1995, período em que o açude tinha um bom volume d'água e em março de 1999, após o completo dessecamento do açude por um período de 3 anos. Utilizou-se para isso um pegador manual com malha de 0,50 $\mathrm{mm}$. As amostras coletadas foram fixadas em formol a 10\%, a triagem através de bandejas iluminadas e a identificação através de estereomicroscópio. Apenas uma espécie de Chironomidae foi registrada, Chironomus gr. decorus, a qual contribuiu com $28,09 \%$ para o total da fauna de insetos aquáticos no mês de agosto/95 e foi a única espécie a colonizar o açude após a seca. No mês de agosto/95 observamos também a ocorrência de Odonata, Coleoptera, Hemiptera, Ephemeroptera e Trichoptera. Neste período constataram-se menores valores para nitrogênio (amônio, nitrito e nitrato), sulfato e condutividade elétrica, comparado com o mês de março/99. Portanto as flutuações, tanto no nível da água assim como nas variáveis físicas e químicas da água do açude Soledade afetam a dinâmica da fauna de insetos aquáticos. Duas considerações principais podem ser apontadas, a partir do aparecimento de Chironomus gr. decorus logo após o período de seca: 1. a oviposição de adultos pode ter sido um importante fator para o processo de recolonização do habitat recém inundado; 2 . outra hipótese a ser considerada, a espécie poderia ter resistido ao período de dessecamento, como larvas no sedimento ou como ovos de resistência.

Palavras-chave: Chironomidae, Chironomus gr. decorus, semi-arido, açude temporário 


\section{Introdução}

Embora as lagoas temporárias sejam comuns em muitos continentes, elas são mais prevalentes e importantes ecologicamente nas regiões áridas e semi-árida onde os recursos d'água permanente pode ser raro ou ausente (Williams, 1985).

$\mathrm{Na}$ América Latina, as lagoas temporárias são ambos usadas tanto como reservatórios d'água nas áreas agrícolas, e como para suplemento de peixe para consumo locais. Estudos de lagoas temporárias podem prover conhecimento sobre sua composição biológica tão bem como as adaptações destes organismos a flutuações sazonais do ambiente (PoncePalafox \& Arredondo-Figueroa, 1998).

A região semi-árida brasileira representa aproximadamente $13,5 \%$ da área total do Brasil, e $74,3 \%$ da região Nordeste (Diniz, 1995), sendo que no estado da Paraíba $99,4 \%$ dos seus municípios sofrem o problema da estiagem.

Os reservatórios ou barragens dessa região sofrem grandes flutuações no nível da água causadas principalmente pela alta taxa de evaporação, altas temperaturas e irregularidade de precipitação, causando impactos sobre a fauna.

Fatores biogeográficos, tais como, idade do corpo d'água, grau de isolamento e a área inundada, e as características do habitat (variedade de substratos, variedade da flora, química da água, predação e competição) são determinantes na composição da fauna de macroinvertebrados em corpos d'água temporários (Friday, 1987). Estes ambientes incluem espécies que são muito bem adaptadas a sobreviver a períodos de seca e que são encontrados exclusivamente em tais habitats, mas também contêm organismos menos adaptados, os oportunistas (Delucchi, 1988).

Muitas larvas de dípteros não possuem adaptações fisiológicas a dessecação e muitos completam a fase larval de seu ciclo de vida antes das lagoas evaporarem (Cantrell \& Mclachlan, 1982). No entanto, há indicações de que alguns quironomídeos sobrevivam longos períodos de dessecação em frestas profundas dentro do substrato (Kaster \& Jacobi, 1978). Outros grupos de insetos ovipõem ovos de resistência (descanso) ou cavam dentro do sedimento e esperam para a lagoa voltar a encher (Skelly, 1997).

Objetivou-se neste trabalho estudar a composição da fauna de Chironomidae e outros insetos aquáticos de um açude temporário e a 
possível influência de algumas variáveis físicas e químicas da água sobre sua abundância. São também discutidas as possíveis estratégias dessa comunidade aquática em sobreviver nestes ambientes.

\section{Material e Métodos}

Característica da área de estudo: $\mathrm{O}$ açude Soledade, fica situado no município de mesmo nome, sobre o planalto da Borborema, nos CaririsVelhos (Caatinga), a $530 \mathrm{~m}$ acima do nível do mar. Sua posição geográfica é 7을' $S$ e 36을 22' W. Represa o riacho Macaco, com alguns pequenos tributários. A construção da barragem foi finalizada em 1933, com uma capacidade de $27.804 .000 \mathrm{~m}^{3}$ e uma área de 541 ha., sendo utilizado para a irrigação e abastecimento d'água do município de Soledade (Melo \& Chacon, 1976). O clima da região é classificado como Bsh (semi-árido quente com chuvas de verão), de 7 a 8 meses seco e 400 a $600 \mathrm{~mm}$ de precipitação/ano. O açude faz parte da Bacia Hidrográfica do Taperoá e os Solos são do tipo salgados alomórficos (Atlas Geográfico do Estado da Paraíba, 1985).

As amostras para análise das variáveis bióticas e da fauna foram coletadas em agosto/1995 e março/1999. No período de novembro/1996 a fevereiro/1999 o açude permaneceu seco devido ao longo período de estiagem.

Variáveis Físicas e Químicas da água: Temperatura da Água $\left({ }^{\circ} \mathrm{C}\right)$ determinada através de um termômetro de mercúrio com $0,5^{\circ} \mathrm{C}$ de precisão; pH - utilizou-se um medidor de pH marca Horiba B-213, digital portátil; Oxigênio Dissolvido $\left(\mathrm{mg} \mathrm{O}_{2} / \mathrm{l}\right)$ foi determinado através do método clássico de Winkler e a Alcalinidade $\left(\mathrm{mgCaCO}_{3} / \mathrm{l}\right)$ - determinada por titulação, ambos através de métodos descritos em Golterman et al., (1978); Condutividade Elétrica $(\mu \mathrm{S} / \mathrm{cm})$ - utilizou-se um conditivímetro Analyser; Dureza Total $(\mu \mathrm{gCaCO} / l)$ - determinada por titulação através do método descrito em Standard Methods (APHA, 1995); O Amônio e o Nitrito foram determinados segundo Mackereth et al. (1978); Nitrato - determinado segundo as técnicas descritas em Rodier (1975).

Fauna de Macroinvertebrados: Foram feitas amostragens qualitativas do zoobentos, utilizando-se um pegador manual com uma rede de malha 
de $0,50 \mathrm{~mm}$. O sedimento coletado foi lavado em peneiras de 1 e 0,20 $\mathrm{mm}$. As amostras foram colocadas em potes plásticos e o material fixado em formol a $10 \%$. No laboratório foi feito a triagem do material em bandejas iluminadas e os indivíduos foram transferidos para frascos de vidro e preservados em álcool a $70 \%$. Os insetos aquáticos foram indentificados seguindo: Merrit \& Cummins (1984) e Trivinho-Strixino \& Strixino (1995).

\section{Resultados e Discussões}

Durante o período de estudo foram registrados 10 taxa para os insetos aquáticos do açude Soledade, Soledade, Paraíba (Tab. I).

Apenas uma espécie de Chironomidae foi registrada, Chironomus gr. decorus, a qual contribuiu com $28,09 \%$ para o total da fauna de insetos aquáticos no mês de agosto/95. Neste mês constatamos a ocorrência de Odonata (Gomphidae, Libellulidae e Coenagrionidae), Coleoptera (Hydrophilidae e Dytiscidae - 23,59 \%) e Hemiptera (Corixidae e Notonectidae - 12,59\%), Baetidae (Ephemeroptera) e Brachycentridae (Trichoptera).

Melo \& Chacon (1976) observou a ocorrência de Chironomidae, Chaoboridae (Chaoborus sp.) e Culicidae no sedimento do açude Soledade, sendo que Chironomidae contribuiu com $91,75 \%$ da fauna de insetos, atingindo uma máxima densidade populacional de $7.111 \mathrm{ind} . / \mathrm{m}^{2}$.

$\mathrm{Na}$ tabela II estão representados os valores das variáveis físicas e químicas da água do açude Soledade. No mês de agosto/95 constatamos menores valores de $\mathrm{pH}(7,3)$, nitrogênio dissolvido na água, amônio $(115.56 \mu / \mathrm{l})$, nitrito $(6.64 \mu / \mathrm{l})$ e nitrato $(475.71 \mu / \mathrm{l})$ e menores valores de sulfato $(3038 \mu / \mathrm{l})$ e de condutividade elétrica $(2390 \mu \mathrm{S} / \mathrm{cm})$, em comparação com o mês de março/99, no qual os valores dessas variáveis, e incluindo a dureza da água $\left(1070 \mathrm{mg} \mathrm{CaCO}_{3} / \mathrm{I}\right)$, foram muito elevados, e de certa maneira pode ter influenciada a composição da fauna de insetos aquáticos.

O aumento nos valores de sais dissolvidos na água do açude Soledade, principalmente refletido nos valores de dureza total e condutividade elétrica, deve estar relacionado ao tipo de solo da região (salgados alomórficos), além da elevada taxa de evaporação da água (Molle, 1994), o que contribui para o aumento da precipitação de sais. 


\section{Tabela I}

Abundância relativa (\%) da fauna de insetos aquáticos do açude Soledade (Soledade, Paraíba) na região semi-árida paraibana.

\begin{tabular}{c|c|c}
\hline Taxa & Agosto/1995 & Março/1999 \\
\hline Diptera & & \\
Chironomidae & 28,09 & 100 \\
Chironomus gr. decorus & 1,12 & \\
Odonata & 4,49 & \\
Gomphidae & 2,25 & \\
Libellulidae & & \\
Coenagrionidae & 6,74 & \\
Ephemerptera & 10,12 & \\
Baetidae & & \\
Trichoptera & 7,87 & \\
Brachycentridae & 23,59 & \\
Coleoptera & 3,37 & \\
Hydrophilidae & 12,36 & \\
Dytiscidae & & \\
Hemiptera & & \\
Corixidae & & \\
Notonectidae &
\end{tabular}

Tabela II

Variáveis físicas e químicas da água do açude Soledade (Soledade, Paraíba) nos meses de agosto de 1995 e março de 1999.

\begin{tabular}{c|c|c}
\hline Variáveis Físicas e químicas & Agosto/95 & Março/99 \\
\hline Temperatura $\left({ }^{\circ} \mathrm{C}\right)$ & 25 & 31.20 \\
Oxigênio Dissolvido $\left(\mathrm{mg} . \mathrm{I}^{-1}\right)$ & 8,02 & 7.98 \\
Condutividade Elétrica $\left(\mu \mathrm{S} . \mathrm{cm}^{-1}\right)$ & 2390 & 4850 \\
$\mathrm{pH}$ & 7.3 & 8.09 \\
Alcalinidade $\left(\mathrm{mg} \mathrm{CaCO} . \mathrm{I}^{-1}\right)$ & 149 & 128 \\
Dureza Total $\left(\mathrm{mg} \mathrm{CaCO}_{3} \cdot \mathrm{I}^{-1}\right)$ & & 1070 \\
Amônio $\left(\mu . \mathrm{I}^{-1}\right)$ & 115.56 & 1882 \\
Nitrito $\left(\mu . \mathrm{I}^{-1}\right)$ & 6.64 & 30.27 \\
Nitrato $\left(\mu . \mathrm{I}^{-1}\right)$ & 475.71 & 1776 \\
Sulfato $\left(\mu . \mathrm{I}^{-1}\right)$ & 3038 & 8105 \\
\hline
\end{tabular}


Com relação as variáveis físicas e químicas do açude Soledade, Melo \& Chacon (1976) observaram concentrações de alcalinidade total de $140,50 \mathrm{mg} / \mathrm{l}$, a dureza total $(473,5 \mathrm{mg} \mathrm{CaCO} / \mathrm{l})$, a concentração hidrogeniônica $(\mathrm{pH})$ apresentou valores constantes de 8,7 e os nutrientes dissolvidos: sulfato $(2360 \mu \mathrm{g} / \mathrm{l})$ e amônio $(25 \mu \mathrm{g} / \mathrm{l})$.

De acordo com Driver (1977) a rápida ou a completa redução no nível da água em lagoas mantém uma comunidade de Chironomidae muito simples, de três a dez espécies. E algumas variáveis químicas da água, tais como o pH e o bicarbonato, mostram relações positivas com a diversidade de Chironomidae. A espécie Chironomus tentans, segundo o autor, eram muito comuns em lagoas semi-permanentes nas quais a condutividade excediam $800 \mathrm{mS} / \mathrm{cm}$. Portanto, valores elevados de condutividasde elétrica, sulfato e de nutrientes dissolvidos do açude Soledade pode ter limitado a colonização de espécies de Chironomidae.

Chironomus gr. decorus foi a única espécie a colonizar o açude Soledade após os 3 anos de seca (novembro/96 a março/99).

Algumas espécies de Chironomidae apresentam alguns mecanismos fisiológicos e comportamentais pelos os quais podem sobreviver a períodos de dessecação do corpo aquático. A partir disso, apontamos algumas considerações, sobre o aparecimento de Chironomus gr. decorus logo após o período de seca:

A recolonização da população de Chironomus gr. decorus no açude Soledade pode ter ocorrido através da oviposição. Chironomus transvaalensis, segundo McLachlan (1969), pode ter recolonizado o lago Kariba (Zambia-Rhodesia) pela a oviposição de fêmeas. McLachlan (1985) observou que Chironomus imicola não era capaz de tolerar a dessecação do habitat, sendo que a recolonização aconteceu pela oviposição.

De acordo com Hinton (1952) larvas de Polypedilum vanderplanki podem resistir a dessecação por um período de 20 meses. Quando o ambiente enche novamente a hidratação das larvas é rápida e estas reassumem as atividades. O mesmo foi observado por McLachlan \& Cantrell (1980) e McLachlan (1983), onde as larvas de P. vanderplanki sobreviveram a frequentes dessecação do seu habitat, sendo capazes de sobreviver como larvas. Assim as precipitações de verão têm uma importante influência na sobrevivência dessas larvas de Chironomidae no substrato exposto (Peterson \& Fernando, 1969). Tal fenômeno não foi observado para o sistema em estudo, mas investigações estão sendo desenvolvidas para verificar se as larvas de Chironomus suportam a dessecação. 
Algumas espécies de Diptera produzem ovos de resistência (Williams, 1987) e de fato, McLachlan (1969) observou que Chironomus transvaalensis, esteve ausente no lago Kariba (Zambia-Rhodesia), exceto durante o período de cheia, sugerindo que pode ter ocorrido a eclosão de ovos de resistência depositados no sedimento. Ainda, algumas espécies de Chironomidae podem sobreviver a períodos de dessecação em cápsulas silicosas e/ou mucosas (Williams, 1987). Embora não tenha sido encontrados ovos de resistência e nem capsulas silicosas estas hipóteses devem ser consideradas e merecem maiores investigações.

No mês de março/99 o açude Soledade se restringia a uma grande poça temporária e além da presença de Chironomus gr. decorus observamos uma grande quantidade de girinos. A presença de girinos aparentemente influencia na velocidade de crescimento das larvas de Chironomidade, reduzindo seu ciclo de vida e o tempo da emergência dos adultos, e aumentando suas chances de emergir antes das lagoas secarem (Cantrell \& McLachlan, 1982). As influências sobre a população de Chironomidae podem se dar através da competição, devido a elevada densidade populacional, pela acumulação de metabólitos e agitação do sedimento pelos girinos (McLachlan, 1985).

\section{Referências Bibliográficas}

APHA, 1995. Standard Methods for examination of water and wastewater. $19^{\circ}$ ed. Washington, American Public, Health Association. 1193 p.

Atlas Geográfico do Estado da Paraíba, 1985. João Pessoa: Secretaria da Educação. 100 p.

Cantrell, M.A. \& Mclachlan, A.J., 1982. Habitat duration and dipteran larvae in tropical rain pools. Oikos 38:343-348

Delucchi, C.M., 1988. Comparison of community structure among streams with different temporal flow regimes. Canadian Jour. Zool. 66:579-586.

Diniz, C.R., 1995. Aspectos sanitários de corpos lênticos temporários para consumo humano. Dissertação de Mestrado, UFPB-Campus II, Campina Grande-PB, 143 p. 
Driver, E.A., 1977. Chironomid communities in small prairie ponds: some characteristics and controls. Freshwater Biol. 7:121-133.

Friday, L.E., 1987. The diversity of macroinvertebrate and macrophyte communites in ponds. Freswater Biol. 18:87-104.

Golterman, H.L.; Clymo, R.S.; Ohnstad, M.A.M., 1978. Methods for physical and chemical analysis of fresh water. $2^{\circ}$ ed. Oxford. Blackwell Sci. Publ. 214 p.

Hinton, H.E., 1952. Survival of chironomid larva after twenty months dehydration. Trans. 9 $^{\text {th }}$ Int. Congr. Entomol. 1:478-482.

Kaster, J.L. \& Jacobi, G.Z., 1978. Benthic macroinvertebrates of a fluctuating reservoir. Freshwater Biol. 8:283-290.

Mackereth, F.J.H., Heron, J. \& Talling, J.F., 1978. Water analysis: some revised methods for limnologists. Dorset, Freshwater Biol. Ass. 121 p.

McLachlan, A.J., 1969. The effect of aquatic macrophytes on the variety and abundance of benthic fauna in a newly created lake in the tropics (Lake Kariba). Arch. Hydrobiol. 66:212-231.

McLachlan, A.J., 1983. Life-history tactics of rain-pool dwellers. J. Anim. Ecol. 52: 545-561.

McLachlan, A.J., 1985. What determines the species present in a rainpool? Oikos 47: 344-351.

McLachlan, A\& Cantrell, M.A., 1980. Survival strategies in tropical rain pools. Oecologia 47:344-351.

Melo, H.A.R. \& Chacon, J.O., 1976. Exame biológico-pesqueiro do açude público "Soledade" (Soledade, PB) Brasil. Bol. Tec. DNOCS 34:3-26.

Merrit, R.W. \& Cummins, K.W., 1984. An introduction to the aquatic insects of North America, $2^{\mathrm{a}}$ ed., Kendall/Hunt Publ. Co. 722 p. 
Peterson, C.G. \& Fernando, C.H., 1969. The macro-invertebrate colonization of a small reservoir in Eastern Canada. Verh. Int. Verein. Limnol. 17:126-136

Ponce-Palafox, J.T. \& Arredondo-Figueroa, J.L., 1998. An analysis of factors governing metabolism of temporary tropical freshwater pond ecosystem. Verh. Int. Verein. Limnol. 26:1571-1574

Rodier, J.,1975. L'analyse de l'eau: eaux naturelles, euax résiduales, eaux de mer. V. 1, 5ed. Dunod (Ed.) Paris. 692 p.

Skelly, D.K., 1977. Tadpole communities: pond permanence and predation are powerful forces shaping the structure of tadpole communities. Am. Scient. 85:36-45.

Trivinho-Strixino, S. \& Strixino, G., 1995. Larvas de Chironomidae (Diptera) do Estado de São Paulo: Guia de identificação e diagnose dos gêneros. PPG-ERN/UFSCar, São Carlos, São Paulo. 229 p.

Williams, D.D., 1987. The ecology of temporary waters. Ed. Croom Helm/ Timber. 193 p.

Williams, W.D., 1985. Biotic adaptations in temporary lentic waters, with special reference to those in semi-arid and arid regions. Hydrobiol. 125:85-110. 